\title{
End-of-day dryness, corneal sensitivity and blink rate in contact lens wearers
}

\author{
Vicente Martín-Montañez ${ }^{\mathrm{a}, \mathrm{b}}$, Alberto López-de la Rosa ${ }^{\mathrm{a}}$, Alberto López-Miguel ${ }^{\mathrm{a}, \mathrm{c}}$, \\ José Pinto-Fraga ${ }^{\mathrm{a}, \mathrm{b}}$, José M. González-Méijome ${ }^{\mathrm{d}}$, María J. González-García ${ }^{\mathrm{a}, \mathrm{b}, *}$ \\ a Ocular Surface Group, IOBA, Universidad de Valladolid, Spain \\ b CIBER-BBN (Biomedical Research Networking Center in Bioengineering, Biomaterials and Nanomedicine), Spain \\ ${ }^{c}$ VISION I+D, SL, Valladolid, Spain \\ d Clinical and Experimental Optometry Research Laboratory, Center of Physics, University of Minho, Braga, Portugal
}

\section{A R T I C L E I N F O}

\section{Article history:}

Received 23 September 2014

Received in revised form 12 January 2015

Accepted 13 January 2015

\section{Keywords:}

Symptoms

Dryness

Corneal sensitivity

Blink rate

Contact lens

\begin{abstract}
A B S T R A C T
Purpose: To study the relationship among the variables intensity of the end-of-day (EOD) dryness, corneal sensitivity and blink rate in soft contact lens (CL) wearers.

Methods: Thirty-eight soft CL wearers (25 women and 13 men; mean age $27.1 \pm 7.2$ years) were enrolled EOD dryness was assessed using a scale of $0-5$ (0, none to 5 , very intense). Mechanical and thermal (heat and cold) sensitivity were measured using a Belmonte's gas esthesiometer. The blink rate was recorded using a video camera while subjects were wearing a hydrogel $\mathrm{CL}$ and watching a film for $90 \mathrm{~min}$ in a controlled environmental chamber.

Results: A significant inverse correlation was found between EOD dryness and mechanical sensitivity $(r:-0.39 ; p=0.02)$; however, there were no significant correlations between EOD dryness and thermal sensitivity. A significant $(r: 0.56 ; p<0.001)$ correlation also was observed between EOD dryness and blink rate, but no correlations were found between blink rate and mechanical or thermal sensitivity.

Conclusions: CL wearers with higher corneal sensitivity to mechanical stimulation reported more EOD dryness with habitual CL wear. Moreover, subjects reporting more EOD dryness had an increased blink rates during wear of a standard CL type. The increased blink rate could act to improve the ocular surface environment and relieve symptoms.
\end{abstract}

(C) 2015 Published by Elsevier Ltd on behalf of British Contact Lens Association.

\section{Introduction}

Despite the large number of contact lens $(\mathrm{CL})$ wearers worldwide [1], discontinuation of wear is still a major problem that limits the amount of successful wearers [2,3], with discomfort and dryness frequently being reported as the main causes [3-5]. The prevalence rates of dryness symptoms among soft CL wearers range between $28 \%$ and $77 \%[2,4,6-8]$. Similar to patients with dry eye disease [9], a substantial number of CL wearers experience symptoms of dryness with CLs and show no clinical signs [10]. This lack of an association between symptoms reported and signs observed is often related to the variability of the clinical tests used to evaluate the signs [11]. However, it also may be due partly to the tests used to evaluate symptoms [12]. Some studies $[4,13]$ have reported dryness changes during the $\mathrm{CL}$ wearing period with increased symptoms

\footnotetext{
* Corresponding author at: IOBA, University of Valladolid, Paseo de Belén 17, 47011 Valladolid, Spain. Tel.: +34 983184756; fax: +34 983184762.

E-mail address: mjgonzalez@ioba.med.uva.es (M.J. González-García).
}

during the afternoon and evening. Hence, symptoms should be assessed at the end of the wearing period to best identify those who may have associated clinical signs, as Begley et al. [13] suggested. In fact, end-of-day (EOD) comfort in CL wearers was evaluated recently and smaller values were reported with silicone hydrogel than with conventional hydrogel CLs [14].

CLs alter the tear film distribution and interact with several parts of the lacrimal functional unit, i.e., the cornea, conjunctiva and eyelids. The sensory nerve terminals of the trigeminal nerve are dense in these tissues, with the cornea the most innervated tissue of all the ocular structures [15] by polymodal nociceptors, mechano-nociceptors, and cold sensitive thermoreceptors. Polymodal-nociceptors are activated by mechanical, heat and chemical stimuli. Mechano-nociceptors (activated by mechanical stimuli) show a relatively higher threshold (lower sensitivity) than the mechanical polymodal nociceptors. Finally, cold thermoreceptors are stimulated when the corneal temperature decreases. Several studies have reported evidence of reduced corneal sensitivity induced by CL wear (soft and rigid CL) [12,16-19]; however, corneal sensitivity seems to be restored after cessation of lens wear 
Table 1

Inclusion and exclusion criteria.

\begin{tabular}{ll}
\hline Inclusion criteria & Age between 18 and 45 years \\
& Spherical refraction between -0.75 and \\
& $-5.00 \mathrm{D}$ and astigmatism $\leq-1.00 \mathrm{D}$ \\
& Best-corrected distance $\mathrm{VA} \leq 0.0$ LogMAR. \\
& Soft CL wear for at least 6 months prior the \\
& study \\
Exclusion criteria & Any active ocular disease \\
& Systemic disease that contraindicates CL wear \\
& Anterior ocular surgery \\
& Use of any topical medication
\end{tabular}

D: diopters; VA: visual acuity; CL: contact lens.

[20]. Nonetheless, little is known about the difference in corneal sensitivity between asymptomatic and symptomatic CL wearers. In fact, one study reported higher corneal responses to suprathreshold stimuli in symptomatic compared to asymptomatic subjects but showed no differences between the two groups in mechanical sensitivity [21].

The blink rate seems to be associated with corneal sensitivity and dryness symptoms; however, the mechanism is unclear. Collins et al. [22] reported a link between corneal sensitivity and blink rate and that the blink rate decreased significantly after administration of topical anesthesia. Other authors $[23,24]$ suggest that the activation of the blink is mediated by tear film disruption sensed by the corneal nerve fibers, while York et al. [25] propose that increased blinking acts to refresh the tear film more frequently and therefore, relieve dryness symptoms.

With these factors in mind, we hypothesized that CL wearers reporting higher ratings of EOD dryness have higher corneal sensitivity and hence, an increased blink rate. The aim of the current study was to evaluate the relationship between EOD dryness levels reported by soft CL wearers during habitual CL wear, basal corneal sensitivity and blink rate. To do this, experiments were conducted while participants wore one single type of hydrogel CL and experienced in the same indoor environment created in a controlled chamber.

\section{Methods}

The nature of the research was explained to the subjects before they provided written informed consent. The study complied with the tenets of the Declaration of Helsinki, and the study protocol was approved by the Institutional Review Board of the University of Valladolid.

Subjects who met the inclusion and exclusion criteria (Table 1) were enrolled. The study was carried out in two visits a minimum of 2 days and a maximum of 15 days apart. Subjects were instructed to not wear CL for at least $24 \mathrm{~h}$ before each visit. During the first visit, the EOD dryness was assessed and the corneal sensitivity was measured. During the second visit, the overall blink rate was measured with the subjects wearing a hydrogel CL (Omafilcon A; CooperVision, Pleasanton, CA) for $90 \mathrm{~min}$ while they were in a controlled standard indoor environment (50\% relative humidity - $\mathrm{RH}, 23^{\circ} \mathrm{C}$ temperature) in an environmental chamber [26,27].

\subsection{Procedures}

End-of-day dryness. Subjects were asked to indicate the intensity of the EOD dryness with habitual CL wear using a scale ranging from 0 to 5 , where 0 meant "none" and 5 meant "very intense." This question was based on the last item of the dryness section of the Contact Lens Dry Eye Questionnaire (CLDEQ) [28], which each participant completed.
Table 2

Descriptive data of tests performed.

\begin{tabular}{lc}
\hline & Mean $\pm \mathrm{SD}$ \\
\hline EOD dryness & $3.32 \pm 1.36$ \\
Corneal sensitivity & $127.89 \pm 41.50$ \\
Mechanical $(\mathrm{ml} / \mathrm{min})$ & $+1.40 \pm 0.87$ \\
Thermal - heat $\left({ }^{\circ} \mathrm{C}\right)$ & $-2.41 \pm 0.95$ \\
Thermal - cold $\left({ }^{\circ} \mathrm{C}\right)$ & \\
Blink rate & $28.25 \pm 15.34$ \\
10 blinks/min & $27.86 \pm 14.83$ \\
30 blinks/min & $29.56 \pm 16.36$ \\
60 blinks/min & $31.38 \pm 16.73$ \\
90 blinks/min & $29.38 \pm 15.23$ \\
Overall value (blinks/min) & \\
\hline
\end{tabular}

EOD: end of day; SD: standard deviation; ml: milliliter; min: minute

Corneal sensitivity. A Belmonte's gas esthesiometer was used as previously described [29-31]. Mechanical and thermal (heat and cold) sensitivity thresholds were measured on the right eye of subjects by the method of levels [29,31]. The mechanical threshold was determined using a controlled airflow ranging between 0 and $200 \mathrm{ml} / \mathrm{min}$ with an airflow temperature reaching the cornea at $34^{\circ} \mathrm{C}$ (the basal corneal temperature) [31]. Then, the thermal thresholds were evaluated using airflow at different temperatures at $10 \mathrm{ml} / \mathrm{min}$ below mechanical threshold to avoid mechanical stimulation. The order of the heat and cold threshold measurements was randomized.

Blink rate. Individuals were fitted with Omafilcon A CL and exposed in an environmental chamber for 90 min under a standard indoor environment [27]. During exposure, the blink rate was recorded using a Live! Cam Socialize HD camera (Creative Technology LTD, Singapore) in primary gaze while subjects were seated watching a film on a 55-inch television (LG Electronics Inc., Gumi, South Korea) $130 \mathrm{~cm}$ above floor level. The blink rate was assessed at four time intervals of $5 \mathrm{~min}(5-10,25-30,55-60$ and $85-90 \mathrm{~min}$ ), and the average blink rate for $1 \mathrm{~min}$ during each interval was obtained [32]. The mean value of the overall exposure was computed for analysis.

\subsection{Statistical analysis}

Statistical analyses were carried out using Statistical Package for the Social Sciences software (SPSS 21.0 for Windows; SPSS Inc., Chicago, IL). EOD dryness, mechanical and thermal corneal sensitivity thresholds, and blink rate values were expressed as the means \pm standard deviations. The normality of the data was assessed with the Shapiro-Wilk test. Analysis of variance was performed to evaluate whether the blink rate differed among the four time intervals. The correlations between variables were calculated using two-tailed Spearman's rho test analysis $(r)$ for nonparametric correlations. A $p$ value $<0.05$ was considered significant.

\section{Results}

Thirty-eight soft CL wearers (25 women and 13 men; mean age $27.1 \pm 7.2$ years; range, 18,45 years) were enrolled. The mean spherical refractive error was $-2.97 \pm 1.12$ diopters (D) (range $-1.25,-4.75$ ), mean cylinder error $-0.27 \pm 0.33 \mathrm{D}$ (range 0.00 , -1.00 ), and mean LogMAR best corrected visual acuity $-0.06 \pm 0.05$ (range $0,-0.19$ ). Subjects had worn CLs for a mean of $7.4 \pm 5.5$ years (range 1,22 ) and wore the CLs for a mean of $9.3 \pm 2.3 \mathrm{~h}$ daily (range 4,18 ).

The descriptive data from the tests of EOD dryness, mechanical and thermal sensitivity, and blink rates are shown in Table 2 . There were no significant $(p=0.78)$ differences in blink rate among the 


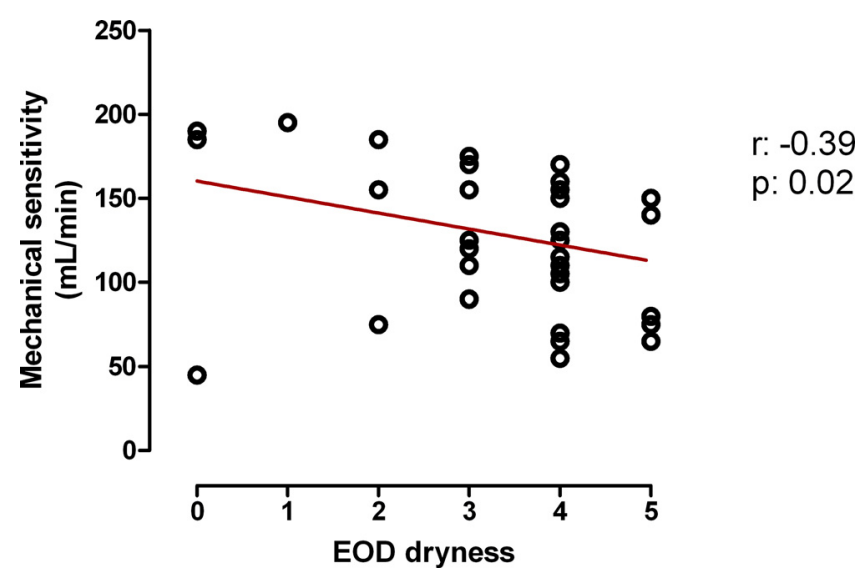

Fig. 1. Correlation between end-of-day (EOD) dryness and mechanical sensitivity.

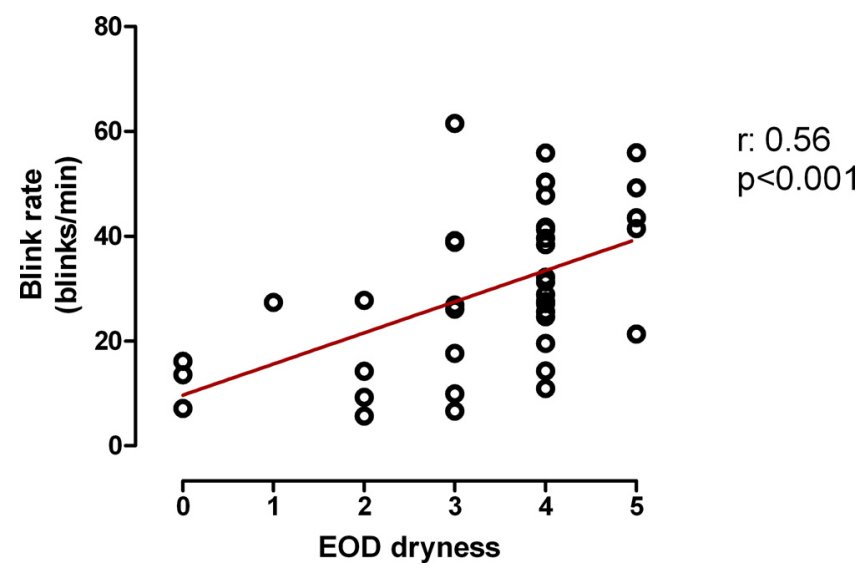

Fig. 2. Correlation between end-of-day (EOD) dryness and blink rate.

four time intervals, and the mean of the four measurements was used to analyze the relationships among variables.

The correlation analysis showed a fair and significant $(r:-0.39$; $p=0.02$ ) inverse correlation between the EOD dryness and mechanical sensitivity (Fig. 1). There were no significant correlations between the EOD dryness and thermal sensitivity. A moderate and significant $(r: 0.56 ; p<0.001)$ positive relationship also was observed between the EOD dryness and blink rate (Fig. 2). However, no correlations were found between the blink rate and mechanical or thermal sensitivity.

\section{Discussion}

CL wearers often use dryness to describe the adverse ocular sensations experienced during wear [33]. Several studies have attempted to identify a connection between clinical signs observed and CL-related discomfort $[11,34]$ to predict and manage the appearance of dryness, which is the primary cause of discontinuation from CL wear $[3,5,4]$. The multifactorial etiology of CL-related dryness makes it difficult to identify indicators that predict its appearance and thus, to develop strategies to avoid discontinuations [10]. Moreover, this lack of an association between signs and symptoms may be related to the questionnaires used to assess the symptoms [12], since dryness can change during the wearing period, with increased symptoms being observed at the EOD. Consequently, some authors have suggested evaluating dryness at EOD, to best identify symptomatic patients [13].

The current results showed an inverse correlation between EOD dryness and mechanical sensitivity in CL wearers. This finding indicated that subjects reporting higher sensitivity to mechanical stimulation are prone to report higher dryness scores at the end of the wearing period with habitual CL wear. Some studies $[35,36]$ have reported sensory adaptation to mechanical stimulation during $\mathrm{CL}$ wear that is mediated by an interaction between the ocular surface and the CL placed on the eye. This interaction may repeatedly stimulate polymodal and mechano-nociceptors, leading to a neural adaptation and thus, decreased corneal sensitivity [37]. Neural adaptation may play a role in the development of CLrelated discomfort, because symptomatic CL wearers do not adapt to suprathreshold mechanical stimuli [21]. Consequently, we suggest that subjects with higher corneal sensitivity are likely to report more symptoms at the end of the wearing time throughout the wearing period, presumably because of decreased ability to develop neural adaptation.

Subjects reporting higher ratings of EOD dryness with habitual CL wear showed higher blink rate during hydrogel CL wear. These results agreed with those previously reported by York et al. [25] who found that CL wearers with dryness symptoms showed an increased blink rate in order to refresh the tear film more frequently. Furthermore, Efron et al. [38] recently suggested that the increased blink rate appears to serve two functions, i.e., to refresh the tear film more often and increase the period of tear film coverage over the ocular surface. Kojima et al. [39] also found that the increased blink rate was not only related to substantial deterioration in symptoms, as in the current study, but also to increased tear osmolarity and tear evaporation rate, reduced tear meniscus volume, and decreased tear film stability. Hence, in the current study, the correlation between the blink rate and dryness symptoms at the end of the wearing time also may be related to other factors that have not been considered such as ocular surface integrity or tear film stability. Thus, further studies including these clinical variables may be interesting.

The numbers of men and women in the current study were not similar. However, the proportion of women recruited was similar to that in a recent study of CL-user demographics in the US, where two-thirds of CL wearers were women [40]. Likewise, subjects' ages in the current study were chosen to be between 18 and 45 years, first because this is similar to the vast majority of CL wearers in the population [40] and second to limit the possible effects of age-related ocular surface changes and near visual symptoms [41] which may have been interpreted in terms of discomfort.

A limitation of the current study was that the EOD dryness and corneal esthesiometry with respect to blink rate assessment were performed during two different visits between 2 and 15 days apart. However, participants were asked about overall EOD dryness based on habitual CL wear, so the questionnaire was not directed specifically to the symptoms on the day of the evaluation [28]. In addition and regarding corneal sensitivity measurements, Teson et al. [30] reported good intersession agreement in corneal sensitivity measured with the Belmonte's esthesiometer.

Another limitation was that EOD dryness was assessed based on subjects' habitual CL, while the blink rate was measured while they wore an omafilcon A CL. It is possible therefore that the measured blink behavior did not accurately reflect that which would have occurred during habitual lens wear, as marked in vitro and in vivo differences in dehydration among several CLs have been reported previously $[26,43,44]$, and these may affect blink behavior. It was, however, considered to be important to standardize the conditions of measurement as much as possible. Consequently all subjects were exposed to the same environmental conditions (50\% relative humidity and $23^{\circ} \mathrm{C}$ temperature, for the same time and fitted with the same CL type).

In summary, the current study identified a link between EOD dryness reported by subjects with their habitual CL wear and corneal mechanical sensitivity. In addition, CL wearers with higher 
EOD dryness ratings seemed to have higher blink rates during wear of the standard omafilcon A CL type. Further studies are required to verify if this behavior reflects that occurring during habitual lens wear, however an increased blink rate may improve the ocular surface environment and therefore relieve symptoms. This research provides further information about the relationship between clinical signs and dryness symptoms in CL wearers by emphasizing the importance of assessing CL-related symptoms at the end of the wearing period.

\section{Conflict of interest}

The authors report no conflicts of interest and have no proprietary interest in any of the materials mentioned in this article.

\section{References}

[1] Morgan P, Woods C, Tranoudis I. International contact lens prescribing in 2011. Contact Lens Spectr 2012;28(January).

[2] Doughty MJ, Fonn D, Richter D, Simpson T, Caffery B, Gordon K. A patient questionnaire approach to estimating the prevalence of dry eye symptoms in patients presenting to optometric practices across Canada. Optom Vis Sci 1997;74:624-31.

[3] Richdale K, Sinnott LT, Skadahl E, Nichols JJ. Frequency of and factors associated with contact lens dissatisfaction and discontinuation. Cornea 2007:26:168-74.

[4] Chalmers RL, Begley CG. Dryness symptoms among an unselected clinical population with and without contact lens wear. Contact Lens Anter Eye 2006;29:25-30.

[5] Young G, Veys J, Pritchard N, Coleman S. A multi-centre study of lapsed contact lens wearers. Ophthalmic Physiol Opt 2002;22:516-27.

[6] Uchino M, Dogru M, Uchino Y, Fukagawa K, Shimmura S, Takebayashi T, et al. Japan ministry of health study on prevalence of dry eye disease among Japanese high school students. Am J Ophthalmol 2008;146:925-9.e2.

[7] Begley CG, Chalmers RL, Mitchell GL, Nichols KK, Caffery B, Simpson T, et al. Characterization of ocular surface symptoms from optometric practices in North America. Cornea 2001:20:610-8.

[8] Uchino M, Nishiwaki Y, Michikawa T, Shirakawa K, Kuwahara E, Yamada M, et al. Prevalence and risk factors of dry eye disease in Japan: Koumi study. Ophthalmology 2011;118:2361-7.

[9] Cuevas M, Gonzalez-Garcia MJ, Castellanos E, Quispaya R, Parra Pde L, Fernández I, et al. Correlations among symptoms, signs, and clinical tests in evaporative-type dry eye disease caused by Meibomian gland dysfunction (MGD). Curr Eye Res 2012;37:855-63.

[10] Dumbleton K, Caffery B, Dogru M, Hickson-Curran S, Kern J, Kojima T, et al. The TFOS international workshop on contact lens discomfort: report of the subcommittee on epidemiology. Invest Ophthalmol Vis Sci 2013;54:TFOS20-36.

[11] Nichols KK, Nichols JJ, Mitchell GL. The lack of association between signs and symptoms in patients with dry eye disease. Cornea 2004;23:762-70.

[12] Stapleton F, Marfurt C, Golebiowski B, Rosenblatt M, Bereiter D, Begley C, et al. The TFOS international workshop on contact lens discomfort: report of the subcommittee on neurobiology. Invest Ophthalmol Vis Sci 2013;54:TFOS71-97.

[13] Begley CG, Caffery B, Nichols KK, Chalmers R. Responses of contact lens wearers to a dry eye survey. Optom Vis Sci 2000;77:40-6.

[14] Papas EB, Tilia D, Tomlinson D, Williams J, Chan E, Chan J, et al. Consequences of wear interruption for discomfort with contact lenses. Optom Vis Sci 2014;91:24-31.

[15] Millodot M. A review of research on the sensitivity of the cornea. Ophthalmic Physiol Opt 1984;4:305-18.

[16] Douthwaite W, Connelly A. The effect of hard and gas permeable contact lenses on refractive error, corneal curvature, thickness and sensitivity. J BCLA 1986;9:14-20.

[17] Bergenske PD, Polse KA. The effect of rigid gas permeable lenses on corneal sensitivity. J Am Optom Assoc 1987;58:212-5.

[18] Situ P, Simpson TL, Jones LW, Fonn D. Effects of silicone hydrogel contact lens wear on ocular surface sensitivity to tactile, pneumatic mechanical, and chemical stimulation. Invest Ophthalmol Vis Sci 2010;51:6111-7.
[19] Murphy PJ, Patel S, Marshall J. The effect of long-term, daily contact lens wear on corneal sensitivity. Cornea 2001;20:264-9.

[20] Millodot M. Effect of the length of wear of contact lenses on corneal sensitivity Acta Ophthalmol (Copenh) 1976;54:721-30.

[21] Chen J, Simpson TL. A role of corneal mechanical adaptation in contact lensrelated dry eye symptoms. Invest Ophthalmol Vis Sci 2011;52:1200-5.

[22] Collins M, Seeto R, Campbell L, Ross M. Blinking and corneal sensitivity. Acta Ophthalmol 1989;67:525-31.

[23] Wolkoff P, Nojgaard JK, Troiano P, Piccoli B. Eye complaints in the office environment: precorneal tear film integrity influenced by eye blinking efficiency. Occup Environ Med 2005;62:4-12.

[24] Collins MJ, Iskander DR, Saunders A, Hook S, Anthony E, Gillon R. Blinking patterns and corneal staining. Eye Contact Lens 2006;32:287-93.

[25] York M, Ong J, Robbins JC. Variation in blink rate associated with contact lens wear and task difficulty. Am J Optom Arch Am Acad Optom 1971;48: $461-7$.

[26] Martín-Montañez V, López-Miguel A, Arroyo C, Mateo ME, González-Méijome $\mathrm{JM}$, Calonge $\mathrm{M}$, et al. Influence of environmental factors in the in vitro dehydration of hydrogel and silicone hydrogel contact lenses. J Biomed Mater Res B Appl Biomater 2014;102(May (4)):764-71.

[27] Tesón M, González-García MJ, López-Miguel A, Enríquez-de-Salamanca A Martín-Montañez V, Benito MJ, et al. Influence of a controlled environment simulating an in-flight airplane cabin on dry eye disease. Invest Ophthalmol Vis Sci 2013;54:2093-9.

[28] Nichols J], Mitchell GL, Nichols KK, Chalmers R, Begley C. The performance of the contact lens dry eye questionnaire as a screening survey for contact lens-related dry eye. Cornea 2002;21:469-75.

[29] Belmonte C, Acosta MC, Schmelz M, Gallar J. Measurement of corneal sensitivity to mechanical and chemical stimulation with a $\mathrm{CO}_{2}$ esthesiometer. Invest Ophthalmol Vis Sci 1999;40:513-9.

[30] Teson M, Calonge M, Fernandez I, Stern ME, Gonzalez-Garcia MJ. Characterization by Belmonte's gas esthesiometer of mechanical, chemical, and thermal corneal sensitivity thresholds in a normal population. Invest Ophthalmol Vis Sci 2012;53:3154-60.

[31] Acosta MC, Tan ME, Belmonte C, Gallar J. Sensations evoked by selective mechanical, chemical, and thermal stimulation of the conjunctiva and cornea. Invest Ophthalmol Vis Sci 2001;42:2063-7.

[32] Cardona G, Garcia C, Seres C, Vilaseca M, Gispets J. Blink rate, blink amplitude, and tear film integrity during dynamic visual display terminal tasks. Curr Eye Res 2011;36:190-7.

[33] Nichols KK, Redfern RL, Jacob JT, Nelson JD, Fonn D, Forstot SL, et al. The TFOS international workshop on contact lens discomfort: report of the definition and classification subcommittee. Invest Ophthalmol Vis Sci 2013;54: TFOS14-9.

[34] Young G, Chalmers R, Napier L, Kern J, Hunt C, Dumbleton K. Soft contact lens-related dryness with and without clinical signs. Optom Vis Sci 2012;89:1125-32.

[35] Polse KA. Etiology of corneal sensitivity changes accompanying contact lens wear. Invest Ophthalmol Vis Sci 1978;17:1202-6.

[36] Lowther GE, Hill RM. Sensitivity threshold of the lower lid margin in the course of adaptation to contact lenses. Am J Optom Arch Am Acad Optom 1968;45:587-94.

[37] Fairhall AL, Lewen GD, Bialek W, de Ruyter Van Steveninck RR. Efficiency and ambiguity in an adaptive neural code. Nature 2001;412:787-92.

[38] Efron N, Jones L, Bron AJ, Knop E, Arita R, Barabino S, et al. The TFOS international workshop on contact lens discomfort: report of the contact lens interactions with the ocular surface and adnexa subcommittee. Invest Ophthalmol Vis Sci 2013;54:TFOS98-122.

[39] Kojima T, Matsumoto Y, Ibrahim OM, Wakamatsu TH, Uchino M, Fukagawa $\mathrm{K}$, et al. Effect of controlled adverse chamber environment exposure on tear functions in silicon hydrogel and hydrogel soft contact lens wearers. Invest Ophthalmol Vis Sci 2011;52:8811-7.

[40] Swanson MW. A cross-sectional analysis of U.S. contact lens user demographics, Optom Vis Sci 2012;89:839-48.

[41] Benjamin WJ, Borish IM. Physiology of aging and its influence on the contact lens prescription. J Am Optom Assoc 1991;62:743-53.

[43] Morgan PB, Efron N. In vivo dehydration of silicone hydrogel contact lenses, Eye Contact Lens 2003;29:173-6.

[44] Gonzalez-Meijome JM, Lopez-Alemany A, Almeida JB, Parafita MA. Dynamic in vitro dehydration patterns of unworn and worn silicone hydrogel contact lenses. J Biomed Mater Res B: Appl Biomater 2009;90:250-8. 